\title{
Exploring instructors' interpretation of electric field lines
}

\author{
Esmeralda Campos ${ }^{1}$ and Genaro Zavala ${ }^{1,2}$ \\ ${ }^{1}$ School of Engineering and Sciences, Tecnologico de Monterrey, Monterrey, Mexico \\ ${ }^{2}$ School of Engineering, Universidad Andres Bello, Santiago, Chile
}

\begin{abstract}
Electric field lines are a typical representation for the electric field concept. University students often misinterpret electric field lines, which may cause them difficulties understanding the electric field. The objective of this study is to explore how introductory electricity and magnetism instructors interpret electric field lines. We interviewed four instructors about several electrostatic concepts, to interpret a diagram and to discuss the electric field line representation. We focus on instructors' interpretation of the diagram, which consisted of the electric field lines of a quadrupole without displaying charges. We asked instructors to interpret the diagram and to compare the magnitude of the electric field at two positions. All four instructors tried to identify the sources of electric field as their first approach, while their answers varied for the comparison of magnitudes. Instructors' interpretations of electric field lines may elucidate why students often misinterpret this representation of the electric field.
\end{abstract}

\section{INTRODUCTION}

The use of representations in physics is one of the concerns of physics education research. Some studies focus on the use of physical representations by experts and novices. Other studies investigate how representations aid in developing students' problem solving skills. A few studies look at how representations help or hinder student understanding of certain physics topics [1].

In introductory courses of Electricity and Magnetism (EM) the concepts are highly abstract and usually new to students. It is necessary to use and translate between different representations to help students develop a robust understanding about electricity and magnetism [1]. The concept of electric field is one of the core concepts in electrostatics. Two typical representations of the electric field are vector field diagrams and electric field lines.

University students often misinterpret electric field lines, which may cause them difficulties to understand the electric field concept. They treat electric field lines as real [2] and as material entities that transport charge [3], confusing electric field lines with trajectories [4]. Students have difficulties finding information about the magnitude of the electric field in electric field lines diagrams [5], applying the principle of superposition and associating the density of lines with the relative magnitude of the field [6].

Instructors have a direct influence on students' views and understanding in Physics [7]. To understand students' interpretation of electric field lines, we believe it is necessary to review instructors' use and interpretation of this representation. The objective of this study is to explore how introductory electricity and magnetism instructors interpret electric field lines.

\section{METHOD}

This report is part of a broader study where we aim to identify why students have difficulties interpreting electric field lines. Exploring how instructors use and interpret electric field lines diagrams will shed some light on this problem. Here we explore instructors' interpretation of electric field lines diagrams. The research questions are: How do electricity and magnetism instructors interpret electric field lines diagrams? How do they find the relative magnitude between two positions in electric field lines diagrams?

\section{A. Participants}

The participants are four instructors of an undergraduate introductory course of Electricity and Magnetism from a private Mexican university. We used the following pseudonyms to respect their confidentiality: Gabriel, Mario, Roberto and Santiago. We chose these instructors because they have very different backgrounds and could bring diverse viewpoints to the table.

Gabriel studied a bachelor and master degree on mechanical engineering and has 24 years of teaching experience. $\mathrm{He}$ has been teaching EM for the last 12 years. Gabriel is the only instructor of the four participants who has worked in industry (15 years). Mario studied a bachelor degree in engineering physics and a master degree in physics. He has seven years of teaching experience and at the time of this study, it was his first semester teaching EM. Roberto studied a bachelor degree in physics and a master degree in engineering. He has 33 years of teaching experience from which most of the time he has been teaching EM. Santiago studied a bachelor and a $\mathrm{PhD}$ degree in physics and has 29 years of teaching experience from which, most of the time, he has been teaching EM.

\section{B. Data collection}

We gathered information using semi-structured interviews. All instructors were interviewed in Spanish. We asked them to explain several concepts as they would to a student, to interpret an electric field lines diagram and to reflect on the importance of electric field line diagrams in different contexts. Here we focus on the interpretation of the electric field lines 


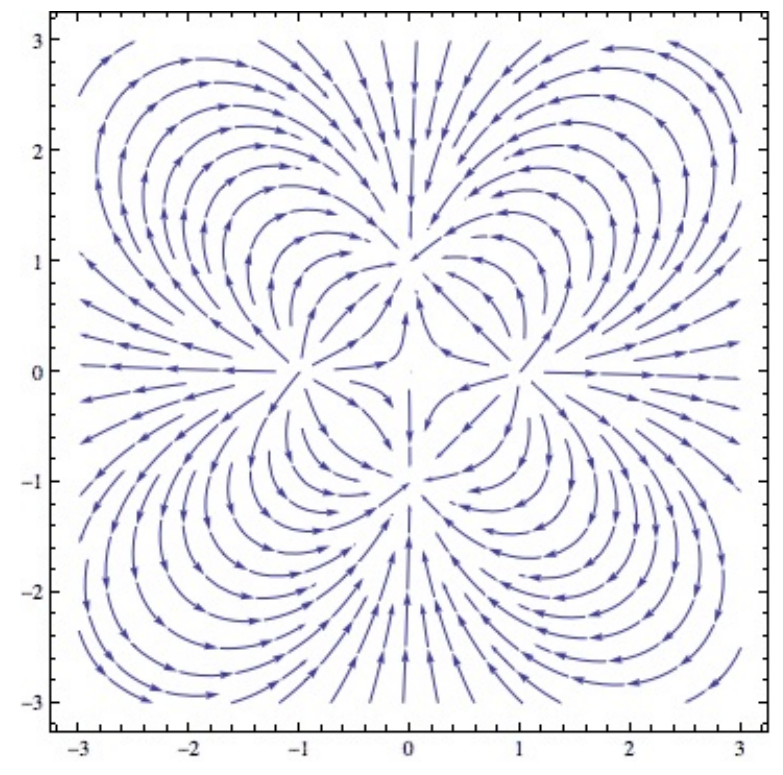

FIG. 1. Diagram of electric field lines of a quadrupole used in the interview. Charges are not shown in the diagram. Image taken from [9]

diagram. The diagram (shown in Fig. 1) consisted of the electric field lines produced by a quadrupole, not showing the distribution of charge.

First, we asked instructors to interpret the diagram. We explicitly told them that it was a free interpretation, and we suggested they used basic concepts of electrostatics (e.g. electric field, electric flux, superposition). We did not specify any particular approach to their interpretation. Second, we asked instructors to compare the magnitude of the electric field at two different positions. We strategically chose two positions where electric field line density was different.

\section{RESULTS}

We analyzed data in three phases. First we transcribed all interviews into spreadsheets, which allowed us to find several keywords in each statement. We grouped together the keywords that expressed the same idea into categories. Then, we created a table where we identified each category, the statement where it was found and the instructor who used it. This organization allowed us to count categories more easily and to identify the frequency per category per professor, as shown in Tables I and II. Since interviews were conducted in Spanish, all transcriptions and the analysis were done in Spanish. The ideas expressed in our results are translations of the original transcription. We present the results for the frequency that each professor expressed each category, as well as the total frequency in two moments: the instructors' first approach and their comparison between two positions.
TABLE I. Instructors' first approach at interpreting an electric field lines diagram (Fig. 1). We identified three predominant categories: Electric field lines leave positive charges, electric field lines arrive to negative charges and the identification of electric field sources.

\begin{tabular}{lccccc}
\hline \hline Categories & G & S & R & M & $\boldsymbol{f}$ \\
\hline Electric field lines leave positive charges & 2 & 2 & 1 & 5 & 10 \\
Electric field lines arrive to negative charges & 2 & 1 & 1 & 4 & 8 \\
Number of field lines that arrive or leave & 1 & & & & 1 \\
Identification of electric field sources & 1 & 2 & 1 & 3 & 7 \\
Distance from the source & & & & 1 & 1 \\
Symmetry & 2 & 1 & 1 & 4 \\
Quadrupole & 1 & & & 1 \\
Test charge & & & & 1 & 1 \\
Repulsion & & & & 3 & 3 \\
Attraction & & & & 1 & 1 \\
Superposition & & & & 1 & 1 \\
\hline \hline
\end{tabular}

\section{A. Instructors' first approach}

When instructors were asked to interpret the electric field lines diagram, all four professors had similar trains of thought. Their first approach was to identify the sources of field. They began by pointing to the areas where they thought there should be a positive charge and stating that electric field lines leave positive charges. They did a similar identification by stating that electric field lines arrive to negative charges. Two instructors referred to positive charges as sources and negative charges as sinks. Table I displays the frequency of occurrence for each category by all instructors.

The most popular category is that "electric field lines leave positive charges", which was stated ten times. This category includes ideas such as: "field lines emerge from there; they are generated there". The second most frequent category is that "electric field lines arrive to negative charges", with eight occurrences. It includes ideas like: "where there is a negative charge there are always arrows pointing towards it". These two categories were usually paired together in a statement. For example, "they (electric field lines) enter negative (charges) and leave positive (charges)". All participants stated these two categories at least once.

The previous categories explain the process of identifying the charges that cause the electric field but are not shown in the diagram. The next category, identification of electric field sources, is the outcome of this thinking process. Here we only considered the moments when instructors explicitly identified charges. It occurred many times implicitly, but it was found explicitly seven times. This category includes ideas like "this is $+q$, this is $+q$, this is $-q$, this is $-q^{\prime \prime}$. All participants stated this category at least once.

We found other categories that we think are relevant to the interpretation of the electric field lines diagram. Some instructors found symmetry within the diagram. Others found 
that field lines represented some interaction, either repulsion or attraction. For instance, they mentioned that the lines going from one source to another represented attraction. Only one instructor mentioned the principle of superposition in his interpretation.

\section{B. Comparison between positions}

When instructors were asked to compare between positions, their responses were quite scattered. The preferred approach was to analyze the distance between the positions and the supposed charges (See Table II, "Distance from the source"). Three out of four instructors, Roberto, Gabriel and Mario, used this approach a total of nine times.

Since the charges were not present in the diagram, we asked instructors to use the resources of the diagram. Gabriel kept on with the category of "Distance from the source" stating ideas like:

"lines are going somewhere, so they are going to a negative charge... where do they come from? From a positive charge... So this (position) is closer to where they are headed, and this is farther away... So from the standpoint of distance this (position) will be more intense than this (position)".

His response suggests that he could not find other resources in the diagram to change his interpretation.

The other two instructors gave very different responses. Roberto chose to talk about the size of the field line, apparently confusing field lines with vectors. On the other hand, Mario expressed that it was necessary to apply the principle of superposition and that electric field lines diagrams when drawn correctly represent the magnitude with the closeness between field lines.

Only one instructor, Santiago, did not compare positions based on the distance from the source. Instead, he tried to approach it by equipotential lines and the angles they made with the supposed charges. Later, he stated that it was not possible to calculate the magnitude of the electric field based solely on the diagram. We asked him if there were any resources in the diagram that could let us compare the magnitudes between two positions. He referred to the closeness between field lines by stating that

\section{"electric field lines represent more intensity when they are closer and less intensity when they are farther".}

\section{DISCUSSION}

Electric field lines diagrams have resources to identify the direction and the relative magnitude of the electric field. The direction of the field is determined by an arrow tangent to the field line, while the magnitude is represented by the density
TABLE II. Instructors were asked to compare the magnitude of the electric field at two positions in an electric field lines diagram without charges. The predominant category was to identify the distance from the source, which is not shown in the diagram. All other categories present a scattered behavior.

\begin{tabular}{lccccc}
\hline \hline Categories & G & S & R & M & $f$ \\
\hline Electric field lines leave positive charges & 2 & & & & 2 \\
Electric field lines arrive to negative charges & 2 & & & & 2 \\
Identification of electric field sources & & & 1 & & 1 \\
Distance from the source & 6 & & 1 & 2 & 9 \\
Symmetry & & 1 & & & 1 \\
Tangent direction & 1 & & & 1 \\
Equipotential lines & 1 & & & 1 \\
More intensity & 2 & & & 2 \\
Field line closeness & 2 & & 1 & 3 \\
Field line size & & & 1 & & 1 \\
Superposition & & & 1 & 1 \\
\hline \hline
\end{tabular}

of lines [8]. In two-dimensional diagrams, the closeness between field lines is enough to represent the density of lines. The diagram may or may not include the charges that produce the electric field. A complete interpretation of the diagram should consider both the direction of the line and the density of field lines or closeness for two-dimensional diagrams.

We observed that instructors' first approach to interpret the electric field lines diagram that we provided was to identify the sources of field. It is interesting to observe this behavior in instructors, as students have shown similar patterns. Students often need to identify the sources of field before making a deeper interpretation [6]. Törnkvist et al. observed that some students tried to identify a source of field in diagrams where they did not include charges [2]. In diagrams where the charges are present, students can more readily apply the principle of superposition, compared to diagrams without the charges [6].

Even though instructors were able to correctly identify the sources of field for this diagram, it is not something that can be done for any distribution of charge. It is more complicated to accurately identify the source in diagrams of electric field lines with continuous or varying distributions of charge and when the distribution is not symmetric. Even in such cases, it would be quite difficult to apply the principle of superposition based only on charges. Students must be able to think about the electric field magnitude and direction when using this representation, even when the charges are not present. For this reason, we believe that it is important for instructors to interpret the density of lines as the relative magnitude of the electric field. We would have expected instructors to have a more holistic view when trying to interpret the electric field diagram that we provided.

In our interviews we observed that instructors do not always use the density of electric field lines as a resource to compare the magnitude of the electric field between posi- 
tions. Instead, they tend to associate the magnitude of the field to the distance between the source of field and the position. Using that distance is not an incorrect approach, but in this case it is not necessary. We believe that instructors tried to use distances to sources because they did not show an integral view of the electric field lines diagram. They were unable to relate the relative magnitude of the electric field to the density of lines. Instead, they tried to use the superposition principle from what they considered were the sources of the field. This is important since there is evidence to support that students have difficulties relating electric field line density with the magnitude of the electric field $[2,5]$. Törnkvist et al. reported that students drew vectors of the same magnitude at different positions in an electric field lines diagram with varying density [2]. Saaraleinen et al. reported that students can identify the magnitude of the field with the distance from the charge, but that they are unable to relate that information to the electric field lines representation [5]. Students think that if a line does not pass through a region there is no electric field in that region [3]. These difficulties may arise when students are not aware of the density of field lines. We think that explicitly relating the magnitude of the field to the density of lines would help students understand and interpret electric field lines correctly.

In the interview, instructors often referred to the convention that electric field lines start at positive charges and end at negative charges. This is something that students use very often [2]. In our interviews, instructors did not use the verbs start or end, but they used expressions like emerge, leave and arrive, which attach certain reality to electric field lines. Students usually think of field lines as real entities [2], and they think that field lines transport or contain charge or force [3]. We believe that, in order to have a complete understanding, it is necessary to navigate between different interpretations. That is, if instructors use those words but understanding that they are used because usually field lines are drawn from positive to negative charges following the arrow in the field line representing the direction of the field tangential to the line at any point, then it is acceptable. However, with that use, students might learn to interpret field lines as real entities, that is, instructors might lead students to think that field lines are actually emerging from a charge or arriving to it. Instructors should consider this in their activities. When introducing electric field line diagrams, instructors should recognize that the way of drawing is just a habit. We think that making explicit through educational activities that electric field lines are not actually emerging or arriving to charges, is important to allow students to understand that they are not real entities.

\section{CONCLUSION}

We asked four instructors of the course of electricity and magnetism to interpret an electric field lines diagram of a quadrupole without the charges present. To answer our two research questions, we analyzed their answers in two parts: the first approach of their interpretation and how they compared the magnitude at two different positions. We observed similarities between how instructors interpret electric field lines and the difficulties that students have understanding this representation. It is important to understand how professors treat electric field lines because we believe that it has a direct influence on student comprehension of this representation.

As their first approach, instructors tried to identify positive and negative charges, a pattern present in students' behavior. This is a correct but not a complete interpretation of the electric field lines diagram, because it does not consider electric field lines density as information regarding the magnitude of the field. When comparing the magnitude of the field at two positions, we found that instructors do not always use the electric field lines' density. Instead, they resorted to the distance between the supposed charges and the positions. Finally, we find that instructors often refer to electric field lines starting at positive charges and ending at negative charges. We discuss how this habit, which could come from drawing lines, may be related to the known difficulty of treating electric field lines as real entities. For each of these findings we suggest instructional activities that could help students overcome their difficulties when interpreting electric field lines.

One limitation of this study is that we interviewed only four instructors, so our results might not be generalizable. Besides, our instructors might not usually reflect on their own use of representations when teaching electricity and magnetism. Interviewing a broader and reflexive community would enrich the discussion and enlighten our understanding of students' difficulties regarding the interpretation of electric field lines.
[1] J. L. Docktor and J. P. Mestre, Phys. Rev. ST Phys. Educ. Res. 10, 020119 (2014), doi: 1554-9178/14/10(2)/020119(58).

[2] S. Törnkvist, K.-A. Pettersson and G. Tranströmer, Am. J. Phys. 61, 4, 335-338 (1993).

[3] M. C. Pocovi and F. N. Finley, Science and Education, 12, 387396 (2003).

[4] Y. Cao and B. M. Brizuela, Phys. Rev. ST Phys. Educ. Res. 12, 020102 (2016), doi: 10.1103/PhysRevPhysEducRes.12.020102.

[5] M. Saarelainen, A. Laaksonen and P. E. Hirvonen, Eur. J. Phys. 28, 51-60, (2007), doi:10.1088/0143-0807/28/1/006.
[6] E. Campos and G. Zavala, in Handbook of Research on Driving STEM learning with Educational Technologies (ed. M. RamirezMontoya), 342-364 (2017).

[7] R. López-Gay and J. Martínez. Enseñanza de las ciencias, 23, 3 (2005).

[8] H. D. Young and R. A. Freedman. University Physics with Modern Physics (13 ed). Addison-Wesley, San Francisco (2013).

[9] http://bolvan.ph.utexas.edu/vadim/classes/15s/ElectricFieldLines.html 